$\mathrm{E}$

EVALUAR
2016, Vol. 16, No 1.

ISSN $1667-4545$

Recuperado de https://revistas.unc.edu.ar/index.php/revaluar

Laboratorio de Evaluación Psicológica y Educativa

Facultad de Psicología - Universidad Nacional de Córdoba

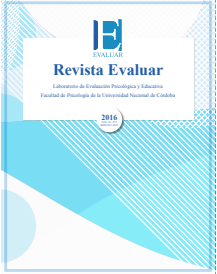

\title{
Datos normativos de la Escala de Procrastinación Académica en estudiantes de psicología de Lima
}

\author{
Sergio Alexis Dominguez-Lara * 1 \\ 1 - Laboratorio Instituto de Investigación de Psicología, Universidad de San Martín de Porres, Lima, Perú.
}

Introducción

Método

Resultados

Discusión

Referencias

\section{Resumen}

El presente trabajo sintetiza la obtención de datos normativos (baremos) de la Escala de Procrastinación Académica (EPA) en estudiantes de psicología de Lima. Participaron 717 estudiantes $(74.3 \%$ mujeres $)$ de edades comprendidas entre 17 y 54 años $(\mathrm{M}=22.65$, DE $=6.49)$. El análisis factorial confirmatorio realizado en la EPA indica que los datos se ajustan a la estructura de dos dimensiones, y los coeficientes de confiabilidad utilizados $(\omega$ y H) presentan magnitudes elevadas. Los puntajes de la EPA no se ajustan a la normalidad, por lo que fueron elaborados baremos utilizando percentiles. Se discuten los resultados y se sugieren guías para utilizar las normas.

Palabras clave: EPA, procrastinación académica, estudiantes universitarios, validez, confiabilidad, normas

\begin{abstract}
This paper synthesizes the normative data obtention (scales) of Academic Procrastination Scale (APS) in Lima psychology students. Seven hundred seventeen students participated (74.3\% women) aged between 17 and 54 years $(\mathrm{M}=22.65, \mathrm{SD}=6.49)$. Confirmatory factor analysis conducted on APS indicates that the data adjust to the structure of two dimensions, and reliability coefficients used ( $\omega$ and $\mathrm{H})$ have high magnitudes. APS scores are not adjusted to normality, so the scales were developed using percentiles. The results are discussed and guidelines are suggested to use the standards.
\end{abstract}

Keywords: emotion, academic procrastination, college students, validity, reliability, scales 


\section{Introducción}

No dejes para mañana lo que puedas hacer hoy, es quizás el producto de la sabiduría popular que mejor refleja el significado de la procrastinación en la vida diaria y en muchas esferas de actuación personal (familia, trabajo, estudios, etc.). Postergar tareas o actividades puede traer como consecuencia que estas se acumulen, sobrecargando a la persona y afectando su salud (Van Eerde, 2003).

En el ámbito universitario, la procrastinación académica (PA) puede definirse como la acción de retrasar voluntaria e innecesariamente la realización de tareas al punto de experimentar malestar subjetivo (Clariana, Cladellas, Badía, \& Gotzens, 2011; Solomon \& Rothblum, 1984), ya sea por falta de agrado hacia la tarea (Steel, 2007), por la dificultad percibida o el miedo a fallar (Brownlow \& Reasinger, 2000; Solomon \& Rothblum, 1984), con consecuencias perjudiciales a mediano y largo plazo (Steel \& Klingsieck, 2016), aunque no existe una definición aceptada universalmente (Schraw, Wadkins, \& Olafson, 2007).

Si bien el componente de dilación de actividades es relevante, al punto de ser el más abordado en los estudios de procrastinación académica (Schraw et al., 2007), el núcleo de la conducta procrastinadora es la falla en los procesos de autorregulación que impiden una adecuada organización y manejo del tiempo, que conduce a postergar los deberes (Balkis \& Duru, 2009; Knaus, 2002; Steel, 2007; Wolters, 2003). Por tal motivo, es lícito pensar que si bien la PA se caracteriza por la postergación, existe un componente relacionado con la conducta académica autorregulada que contribuye a la PA (Grunschel, Patrzek, \& Fries, 2013; Klingsieck, Grund, Schmid, \& Fries, 2013; Steel \& Klingsieck, 2016).

Existen diversos instrumentos para evaluar la PA (Aitken, 1982; Solomon \& Rothblum, 1984; Lay, 1986; Tuckman, 1991), e inclusive la ATPS (Tuckman, 1991) ha sido trabajada en idioma español (Furlan, Heredia, Piemontesi, \& Tuckman, 2012). Sin embargo, todos ellos conciben de forma diferenciada la dimensionalidad de la PA, ya que dos de ellos la consideran como un constructo unidimensional, enfocado en la evitación de la tarea (Tuckman, 1991) o la medición de la postergación (Lay, 1986); mientras que dos de ellos la consideran como formada por dos dimensiones: miedo a fallar y evitación de la tarea (Aitken, 1982; Solomon \& Rothblum, 1984).

En este sentido, la Escala de Procrastinación Académica (EPA; Busko, 1998), debido a su brevedad (12 ítems) y su estructura, parece ser una buena opción para su evaluación. La EPA (originalmente con 16 ítems), fue construida para ser utilizada con estudiantes universitarios, y consideró a la PA como la tendencia a dejar de lado las tareas (Busko, 1998). Álvarez-Blas (2010) tradujo su contenido al español hablado en Perú, y fue empleada en población escolar limeña. En ambos estudios (Álvarez-Blas, 2010; Busko, 1998) la PA es considerada como unidimensional, pero en una investigación realizada en universitarios de Lima se halló una estructura de dos dimensiones: Postergación de actividades y Autorregulación académica, aunque con baja correlación entre sí (Dominguez-Lara, Villegas-García, \& CentenoLeyva, 2014), y sin evidencias suficientes para plantear la existencia de un factor de segundo orden que justifique la sumatoria de las dos puntuaciones.

Esta independencia no es incoherente, porque haría referencia a dos procesos separados, e indicaría que un estudiante puede postergar sus actividades, pero a su vez planificar, por ejemplo, cómo y cuándo las hará; o, inclusive, una persona podría no dilatar la ejecución de ciertas tareas, y aun así poseer baja capacidad para organizar 
sus actividades. El caso más extremo sería que presente alta postergación de actividades y baja autorregulación académica. Todos esos casos son habituales en las aulas universitarias, por lo que es necesario establecer perfiles que permitan identificar la problemática de cada estudiante en torno a la PA, y de ese modo plantear intervenciones efectivas.

La prevalencia de PA entre los universitarios es elevada (Steel, 2007). A nivel internacional se observan tasas entre el $10.2 \%$ y $75 \%$ (Balkis \& Duru, 2009; Brownlow \& Reasinger, 2000; Ellis \& Knaus, 1977; Özer, 2011; Özer, Demir, \& Ferrari, 2009; Potts, 1987; Solomon \& Rothblum, 1984), mientras que en Perú, el 14.1\% de la muestra estudiada presenta PA (DominguezLara, en revisión a), aunque los hallazgos se focalizaron sólo en la dimensión relacionada con la postergación de actividades.

Por tal motivo, es necesario obtener valores normativos de la EPA que faciliten su uso en evaluaciones masivas para detectar estudiantes con un potencial perfil de riesgo. No obstante, en este tipo de estudios el punto de corte elegido para la configuración de perfiles tiende a ser objeto de controversia.

En investigaciones enfocadas en la prevalencia de PA los métodos de clasificación procrastinador / no procrastinador suelen ser heterogéneos. Algunos se enfocan en respuestas extremas. Por ejemplo, si en una escala del uno al cinco elige una respuesta $\geq 4$ (Harriot \& Ferrari, 1996; Solomon \& Rothblum, 1984); mientras que otros clasifican como procrastinadora a una persona si puntúa por encima de la mediana (Brownlow \& Reasinger, 2000; Özer, 2011; Özer et al., 2009); o si su puntuación es mayor a una desviación estándar (DE) por encima de la media (Balkis \& Duru, 2009). Según un estudio preliminar (Dominguez-Lara, en revisión a), el tercer método es más efectivo ya que evita los falsos positivos al centrarse en las puntuaciones más extremas. Además, utilizando el tercer método, la diferencia entre procrastinadores y no-procrastinadores en cuanto a la autoeficacia académica evidencia puntuaciones más elevadas en el segundo grupo, y el coeficiente $K 2$ (Livingston, 1972) para evaluar la confiabilidad del punto de corte es más elevado respecto a los métodos anteriores. Sin embargo, ese procedimiento fue empleado sólo en la dimensión de postergación de la EPA, y la clasificación considerada fue dicotómica. En ese sentido, y siguiendo la línea de análisis aplicada a otros estudios para la obtención de baremos (Dominguez-Lara, en revisión b), serán considerados percentiles (Pc) para establecer los puntos de corte.

Por lo tanto, los objetivos de esta investigación fueron: 1) analizar la estructura interna y confiabilidad de las puntuaciones de la EPA, 2) brindar datos normativos de las dimensiones de la EPA, 3) evaluar la confiabilidad de los puntos de corte elegidos, y 4) analizar la confiabilidad del perfil.

\section{Método}

\section{Participantes}

La muestra intencional estuvo formada por 717 estudiantes universitarios de psicología (74.3\% mujeres; $33.5 \%$ trabaja; $92.2 \%$ es soltero) de tres universidades privadas de Lima Metropolitana $\left(\mathrm{n}_{1}=280 ; \mathrm{n}_{2}=346, \mathrm{n}_{3}=91\right)$ que cursaban entre primer y décimo ciclo de estudios. No se observaron diferencias en cuanto a la distribución de varones y mujeres según universi$\operatorname{dad}\left(\chi_{[2]}^{2}=3.706, p=.157, V=.072\right)$. El rango de edad estuvo fue de entre 17 y 54 años $(\mathrm{M}=$ 22.65, DE $=6.49)$, sin presentar diferencias entre varones y mujeres $\left(t_{(715)}=1.149, p=.251\right)$. 
Instrumentos

Escala de Procrastinación Académica (EPA; Busko, 1998). Fue utilizada la adaptación a universitarios limeños (Dominguez-Lara et al., 2014) que consta de dos dimensiones: Postergación de actividades (tres ítems) y Autorregulación académica (nueve ítems). Los ítems cuentan con cinco opciones de respuesta (Nunca, Pocas veces, $A$ veces, Casi siempre, Siempre). La interpretación de los puntajes es directa: a mayor puntaje, mayor presencia de la conducta evaluada.

\section{Procedimiento}

La aplicación de la EPA fue realizada durante los horarios de clase. Previamente fueron explicados los objetivos de la investigación resaltando el carácter voluntario de la participación, y que esta no sería recompensada académica o económicamente. Sólo fueron evaluados quienes brindaron su consentimiento verbal.

\section{Tratamiento estadístico de los datos}

En cuanto al análisis estructural, previo análisis descriptivo de los ítems, fue evaluada la dimensionalidad (modelo unidimensional, y de dos factores oblicuos) de la EPA mediante un análisis factorial confirmatorio (AFC) con el programa EQS 6.2 (Bentler \& Wu, 2012) bajo las siguientes condiciones: método de máxima verosimilitud, matriz de correlaciones policóricas (Lee, Poon, \& Bentler, 1995; Dominguez-Lara, 2014) y estadísticos robustos. El ajuste del modelo fue evaluado utilizando la prueba SB- $\chi^{2}$ (Satorra \& Bentler, 1994), RMSEA ( $\leq .05)$, CFI $(\geq .95)$, y el SRMR $(\leq .08)$ de forma conjunta.

Con respecto al análisis de la confiabilidad, fueron empleados los coeficientes $\omega$ (McDonald, 1999) y $H$ (Dominguez-Lara, 2016b; Hancock \& Mueller, 2001), debido a que son más adecuados ante el incumplimiento del supuesto de tau-equivalencia de los ítems (Dominguez-Lara, 2016a).

Para la elaboración de baremos fue replicado el procedimiento de un estudio previo (Dominguez-Lara, en revisión b): análisis de normalidad con la prueba de Shapiro-Wilk (Ghasemi \& Zahedias1, 2012), cálculo de diversos Pc (5, $10 . .99$ ), con énfasis en los Pc 25 y 75 para delimitar los puntos de corte de los niveles Alto ( $>$ Pc 75) y Bajo ( < Pc 25) en cada dimensión evaluada y el cálculo del coeficiente $K 2$ para la confiabilidad del punto de corte (Fernández-Arata \& Merino-Soto, 2014; Livingston, 1972).

De forma adicional, fue evaluada la confiabilidad de la diferencia entre puntuaciones $\left(\rho_{\mathrm{d}}\right)$ al interior del perfil (Muñiz, 2003; DominguezLara, 2016c). Este coeficiente va desde cero a la unidad, y magnitudes elevadas de $\rho_{\mathrm{d}}$ indican que la diferencia entre puntajes se debe más a la varianza verdadera, que a la varianza del error. Finalmente, fue calculada la diferencia mínima confiable $(D)$, es decir, la diferencia mínima esperada entre las dos puntuaciones que no es atribuible al error de medición (Charter, 1996; Dominguez-Lara, Merino-Soto, \& NavarroLoli, 2016). Por ejemplo, si $D$ es 15 , y el valor absoluto de la diferencia entre las puntuaciones de las dos dimensiones es $10\left(\left|\mathrm{P}_{1}-\mathrm{P}_{2}\right|=10\right)$, esa diferencia es producto del error de medición. En ambos casos, si la magnitud de $\rho_{\mathrm{d}}$ no es satisfactoria y $\left|\mathrm{P}_{1}-\mathrm{P}_{2}\right|<D$, no sería recomendable usar ese perfil para consideración individual.

\section{Resultados}

En cuanto a las características descriptivas de los ítems, las respuestas tienden a la 
Tabla 1

Estadísticos descriptivos de los ítems de la EPA.

\begin{tabular}{|c|c|c|c|c|c|}
\hline Ítem & $\mathbf{M}$ & DE & $\mathrm{g}_{1}$ & SSI & $\mathbf{g}_{2}$ \\
\hline $\begin{array}{l}\text { Cuando tengo que hacer una tarea, normalmente la dejo para el último } \\
\text { minuto.* }\end{array}$ & 2.891 & .893 & -.174 & .109 & .190 \\
\hline 2. Generalmente me preparo por adelantado para los exámenes. & 3.018 & .858 & -.048 & .033 & -.172 \\
\hline $\begin{array}{l}\text { 3. Cuando tengo problemas para entender algo, inmediatamente trato de } \\
\text { buscar ayuda. }\end{array}$ & 3.452 & 1.073 & -.232 & .101 & -.559 \\
\hline 4. Asisto regularmente a clase. & 3.816 & 1.385 & -.964 & .250 & -.400 \\
\hline 5. Trato de completar el trabajo asignado lo más pronto posible. & 3.572 & 1.126 & -.562 & .222 & -.345 \\
\hline 6. Postergo los trabajos de los cursos que no me gustan.* & 2.544 & .987 & .078 & .040 & -.476 \\
\hline 7. Postergo las lecturas de los cursos que no me gustan.* & 2.596 & 1.013 & .139 & .068 & -.442 \\
\hline 8. Constantemente intento mejorar mis hábitos de estudio. & 3.428 & 1.037 & -.452 & .210 & -.337 \\
\hline $\begin{array}{l}\text { 9. Invierto el tiempo necesario en estudiar aún cuando el tema sea } \\
\text { aburrido. }\end{array}$ & 3.244 & .946 & -.047 & .026 & -.393 \\
\hline 10. Trato de motivarme para mantener mi ritmo de estudio. & 3.492 & 1.119 & -.547 & .218 & -.366 \\
\hline 11. Trato de terminar mis trabajos importantes con tiempo de sobra. & 3.391 & .968 & -.263 & .141 & -.245 \\
\hline 12. Me tomo el tiempo de revisar mis tareas antes de entregarlas. & 3.526 & 1.107 & -.472 & .193 & -.411 \\
\hline
\end{tabular}

Nota. $\mathrm{n}=717$; *: Pertenecen a la dimensión Postergación de actividades; M: media aritmética. DE: desviación estándar; g: asimetría de Fisher; SSI: Índice estandarizado de asimetría; $\mathrm{g}_{2}$ : curtosis de Fisher.

opción intermedia en los ítems de la dimensión Postergación de actividades, mientras que en los de Autorregulación académica se orientan hacia la opción más elevada (Tabla 1). En ambos casos, su dispersión está dentro de los límites tolerables (SSI $\leq .25$; Malgady, 2007).

Con relación a la evaluación de la dimensionalidad, el modelo unidimensional (12 ítems influidos por un solo constructo) mostró un ajuste desfavorable: SB- $\chi_{(54)}^{2}=626.519(p<.001)$, CFI $=.904$, RMSEA $($ IC 90\%) =.122 (.113 - .130), $\mathrm{SRMR}=.124$. Por el contrario, el ajuste del modelo de dos factores oblicuos fue adecuado: $\mathrm{SB} \chi_{(53)}^{2}=204.976(p<.001), \mathrm{CFI}=.974$, RMSEA (IC 90\%) =.063 (.054 - .072), SRMR $=.071$. Así mismo, en promedio, todas las cargas factoriales fueron de magnitud moderada a alta $(\lambda$ postergación $=.751 ; \lambda$ regulación $=.683)$ y esta- dísticamente significativas $(p<.001)$. No obstante, la correlación entre factores fue bastante baja $(\phi=-.118)$.

Finalmente, los coeficientes de confiabilidad fueron elevados tanto en la dimensión Postergación de actividades $(\omega=.811 ; H=.894)$ como en Autorregulación académica $(\omega=.892$; $H=.914$; Hancock \& Mueller, 2001; MerinoSoto, Navarro-Loli, \& García-Ramirez, 2014).

\section{Datos normativos}

De forma previa a la elaboración de datos normativos, fueron evaluadas las características distribucionales de la escala. La puntuación de la EPA no se aproxima a una distribución normal tanto en Postergación de actividades (SW 
$\left.{ }_{[717]}=.975, p<.001\right)$ como en Autorregulación académica $\left(S W_{[717]}=.978, p<.001\right)$. Para ambas dimensiones, los puntajes por encima del Pc 75 y por debajo del Pc 25 indican los niveles alto y bajo, respectivamente (Tablas 2 y 3 ). De forma práctica, en cuanto a Postergación de actividades, puntuaciones menores que siete indicarían un nivel bajo, y mayores que nueve, nivel alto; mientras que en Autorregulación académica, puntuaciones por debajo de 27 indicarían un nivel bajo, y por encima de 35 , nivel elevado.

Tabla 2

Datos normativos de la EPA en estudiantes de psicología: Postergación de actividades.

\begin{tabular}{|c|c|c|}
\hline$P D$ & Pc & $K 2$ \\
\hline $3-4$ & 5 & $.955-.937$ \\
\hline 5 & 10 & .907 \\
\hline 6 & 20 & .858 \\
\hline 7 & 25 & .793 \\
\hline 8 & 40 & .754 \\
\hline 9 & 55 & .789 \\
\hline 10 & 80 & .854 \\
\hline 11 & 90 & .904 \\
\hline $12-13$ & 95 & $.935-.954$ \\
\hline 14 & 99 & .966 \\
\hline$M$ & \multicolumn{2}{|c|}{8.03} \\
\hline$D E$ & \multicolumn{2}{|c|}{2.372} \\
\hline$g_{1}$ & \multicolumn{2}{|c|}{.006} \\
\hline$g_{2}$ & \multicolumn{2}{|c|}{-.017} \\
\hline
\end{tabular}

Nota. PD: Puntuación directa; Pc: Percentil; K2: Coeficiente K2; $M$ : media aritmética. $D E$ : desviación estándar; $g_{1}$ : asimetría de Fisher; SSI: Índice estandarizado de asimetría; $g_{2}$ : curtosis de Fisher.

Los coeficientes $K 2$ mostraron consistentemente puntuaciones elevadas $(>.90)$, la confiabilidad de las diferencias $\left(\rho_{\mathrm{d}}=.867\right)$ es de magnitud aceptable, y la $D$ requerida a un $95 \%$ de confianza es de 12 puntos. Es decir, si la di- ferencia es menor que 12 , se interpretaría como efecto del error de medición. Respecto a esto último, del total de evaluados sólo el $6.4 \%$ presentó diferencias atribuibles al error de medición $\left(\mid \mathrm{P}_{1}\right.$ $-\mathrm{P}_{2} \mid<D$ ). Por ello, el perfil que se configure a partir de la EPA tiene evidencias favorables para ser considerado de forma integral (Tabla 3).

Entonces, en vista de que es viable la consecución de un perfil confiable, se realizó una distribución de frecuencias en una tabla de contingencia con relación a los niveles de cada dimensión, a fin de conocer qué porcentaje poseía el perfil de riesgo descrito anteriormente: baja autorregulación y elevada postergación. De acuerdo con los hallazgos, 46 personas $(6.42 \%)$ son caracterizadas por dicho perfil. Asimismo, otros 103 estudiantes (14.37\%) estarían cercanos a esa situación (Tabla 4).

\section{Discusión}

Con los hallazgos del presente estudio se aporta mayor evidencia respecto a la dimensionalidad de la EPA, es decir, a la configuración planteada en un estudio preliminar (DominguezLara et al., 2014). En ese sentido, la EPA evalúa de forma confiable los dos aspectos clave en la conducta procrastinadora: la Postergación de actividades, y la Autorregulación académica; de los cuales fue posible obtener valores normativos aplicables a estudiantes de psicología.

El principal aporte práctico del estudio es la consideración de los dos puntajes independientes de la EPA (uno por cada dimensión) estructurados en un perfil que permite una aproximación a la conducta académica del estudiante. El perfil cuenta con evidencia favorable y facilitaría una identificación más rápida de aquellos alumnos que necesitan algún tipo de orientación, como el caso de los estudiantes con nivel alto de 
Tabla 3

Datos normativos de la EPA en estudiantes de psicología: Autorregulación académica.

\begin{tabular}{|c|c|c|c|c|c|}
\hline$P D$ & Pc & $K 2$ & $P D$ & $P c$ & $K 2$ \\
\hline $12-18$ & 5 & $.985-.971$ & 32 & 50 & .871 \\
\hline $19-21$ & 10 & $.968-.958$ & 33 & 60 & .879 \\
\hline $21-24$ & 15 & $.951-.936$ & 34 & 65 & .890 \\
\hline $25-26$ & 20 & $.925-.913$ & 35 & 75 & .903 \\
\hline 27 & 25 & .901 & $36-37$ & 80 & $.915-.927$ \\
\hline 28 & 30 & .889 & 38 & 85 & .937 \\
\hline 29 & 35 & .878 & 39 & 90 & .945 \\
\hline 30 & 40 & .870 & $40-41$ & 95 & $.953-.959$ \\
\hline 31 & 45 & .868 & $42-44$ & 99 & $.964-.975$ \\
\hline$M$ & \multicolumn{2}{|c|}{30.94} & & & \\
\hline$D E$ & \multicolumn{2}{|c|}{6.772} & & & \\
\hline$g_{1}$ & \multicolumn{2}{|c|}{-.481} & & & \\
\hline$g_{2}$ & \multicolumn{2}{|c|}{-.076} & & & \\
\hline
\end{tabular}

Nota. PD: Puntuación directa; Pc: Percentil; K2: Coeficiente K2; $M$ : media aritmética. $D E$ : desviación estándar; $g_{1}$ : asimetría de Fisher; SSI: Índice estandarizado de asimetría; $g_{2}$ : curtosis de Fisher.

Tabla 4

Relación entre los niveles de Postergación de actividades y Autorregulación académica.

\begin{tabular}{lrcccc}
\hline & & \multicolumn{2}{c}{ Nivel de Autorregulación académica } & \multirow{2}{*}{ Total } \\
\cline { 3 - 5 } & & Bajo & Promedio & Alto & \\
\hline & Bajo & 43 & 57 & 76 & 176 \\
Nivel de Postergación & Promedio & 80 & 212 & 80 & 372 \\
de Actividades & Alto & 46 & 103 & 20 & 169 \\
& Total & 169 & 372 & 176 & 717 \\
\hline
\end{tabular}

Postergación, pero bajo en Autorregulación, permitiendo de ese modo evitar el abandono o fracaso académico (González-Brignardello \& Sánchez-Elvira-Paniagua, 2013), además de las consecuencias emocionales que trae consigo la PA (p. ej., Steel \& Klingsieck, 2016). No obstante, dicha clasificación se basa en la distribución de puntajes de la muestra, por lo que sería conveniente realizar estudios independientes a fin de comparar los grupos con respecto a atributos psicológicos vinculados con la PA como la ansiedad ante exámenes (Quant \& Sánchez, 2012), auto- eficacia académica (Wäschle, Allgaier, Lachner, Fink, \& Nückles, 2014) y ansiedad (Klassen, Krawchuk, \& Rajani, 2008; Pardo-Bolívar, Perilla-Ballesteros, \& Salinas-Ramírez, 2014), entre otros. En esas circunstancias, sería esperable un desempeño más adaptativo en los grupos más orientados a la autorregulación y menos a la postergación.

Por ejemplo, la consideración de cada puntaje de forma independiente puede ayudar a una visualización más precisa de algunos procesos recurrentes entre los universitarios, como la 
naturaleza de la ansiedad ante exámenes (AE; Spielberger \& Vagg, 1995) que presenten los estudiantes. Existen planteamientos acerca del tipo de AE que el estudiante experimenta según el grado de organización de su conducta académica. Es decir, existiría un tratamiento diferenciado para la persona que experimenta elevada AE y posee una conducta académica organizada así como el conocimiento necesario para aprobar el examen (AE irracional); y un tipo de orientación distinta para el individuo que no es capaz de planificar su tiempo antes de una evaluación $\mathrm{y}$ posee un conocimiento precario del curso, cuya AE sería producto de esa carencia (AE racional; Naveh-Benjamin, McKeachie, \& Lin, 1987; Serrano-Pintado \& Escolar-Llamazares, 2014; Serrano-Pintado, Sánchez-Mateos, \& Escolar-Llamazares, 2010). Por ello, los datos normativos de la EPA, de forma conjunta con otros hallazgos (Dominguez-Lara, en revisión c), podrían ayudar a clasificar a los estudiantes según su Autorregulación y AE experimentadas, orientando de manera más precisa su potencial tratamiento (Serrano-Pintado, Sánchez-Mateos, \& Escolar-Llamazares, 2016) en el marco de políticas institucionales orientadas al bienestar del estudiante. Cabe precisar que los puntos de corte elegidos en algunas ocasiones son arbitrarios, ya que no existe una norma general que indique cuál es el método más apropiado, aunque es una preocupación constante en la comunidad científica generar puntos de corte que permitan reducir al máximo el error, sobre todo en situaciones donde las decisiones tendrán repercusión directa en el evaluado (Ramírez-Benítez, Jiménez-Morales, \& Díaz-Bringas, 2015). En el caso concreto de este estudio, si bien el procedimiento escogido (percentiles, centiles, cuartiles) es ampliamente usado (p. ej., Sánchez-Rosas, 2015) y las clasificaciones hechas en torno a los cuartiles brindan una mayor probabilidad de que los grupos estén bien diferenciados, la interpretación de los resultados debe realizarse con cautela. Por ello, los hallazgos a partir de la EPA (y de cualquier otro instrumento) deben ser contrastados con una entrevista $\mathrm{y}$, si fuera posible, complementados con otras fuentes de información (p. ej., record académico). La limitación principal del estudio no es el tamaño muestral, debido a que otros trabajos de estandarización usaron muestras de similar magnitud (Furlan, Pérez, Moyano, \& Cassady, 2010) y los resultados fueron alentadores; aunque sí podría serlo el tipo de muestreo (intencional). Probablemente el hecho de contar con una sola carrera profesional limite su uso sólo a estudiantes de psicología. Por tal motivo, es recomendable ampliar las aplicaciones a otras carreras profesionales, ya que de ese modo se podrá tener una visión más amplia de la conducta académica de los estudiantes en diferentes escenarios, con diferentes exigencias, ya que existe evidencia de que no se postergan todas las tareas por igual (Blunt \& Pychyl, 2000). A su vez, realizar un muestreo aleatorio permitirá aproximarse más precisamente al comportamiento poblacional y de ese modo, generalizar con un mayor grado de confianza.

\section{Referencias}

Aitken, M. E. (1982). A personality profile of the college student procrastinator (Tesis doctoral inédita). University of Pittsburgh, Estados Unidos.

Álvarez-Blas, O. R. (2010). Procrastinación general y académica en una muestra estudiantes de secundaria de Lima metropolitana. Persona, 13, 159-177. Recuperado de http://www.redalyc.org/revista.oa?id=1471

Balkis, M., \& Duru, E. (2009). Prevalence of academic procrastination behavior among pre-service teachers, and its relationship with demographics and individual preferences. Journal of Theory and Practice in Education, 5(1), 18-32. Recuperado de http://eku. 
comu.edu.tr/index

Bentler, P. M., \& Wu, E. J. C. (2012). EQS 6.2 for Windows [Statistical Program]. Encino, CA: Multivariate Software, Inc.

Blunt, A. K., \& Pychyl, T. A. (2000). Task aversiveness and procrastination: A multi-dimensional approach to task aversiveness across stages of personal projects. Personality and Invididual Differences, 28(1), 153167. doi: 10.1016/S0191-8869(99)00091-4

Brownlow, S., \& Reasinger, R. D. (2000). Putting off until tomorrow what is better done today: Academic procrastination as a function of motivation toward college work. Journal of Social Behavior and Personality, 15(5), 15-34.

Busko, D. A. (1998). Causes and consequences of perfectionism and procrastination: A structural equation model (Tesis de Maestría inédita). The University of Guelph, Canada.

Charter, R. A. R. (1996). Formulas for reliable and abnormal differences in raw test scores. Perceptual and Motor Skills, 83(3), 1017-1018. doi: 10.2466/ pms.1996.83.3.1017

Clariana, M., Cladellas, R., Badía, M. del M., \& Gotzens, C. (2011). La influencia del género en variables de la personalidad que condicionan el aprendizaje: inteligencia emocional y procrastinación académica. Revista Electrónica Interuniversitaria de Formación del Profesorado, 14(3), 87-96. Recuperado de http:// www.aufop.com/aufop/revistas/lista/digital

Dominguez-Lara, S. A. (2014). ¿Matrices Policóricas/Tetracóricas o matrices Pearson? Un estudio metodológico. Revista Argentina de Ciencias del Comportamiento, 6(1), 39-48. Recuperado de https://revistas. unc.edu.ar/index.php/racc/index

Dominguez-Lara, S. A. (2016a). Secretos del coeficiente alfa. Actas Urológicas Españolas, 40(7), 471. doi:10.1016/j.acuro.2016.04.002

Dominguez-Lara, S. A. (2016b). Evaluación de la confiabilidad del constructo mediante el Coeficiente $\mathrm{H}$ : Breve revisión conceptual y aplicaciones. Psychologia: Avances en la disciplina, 10(2), 87-94. doi:
$10.21500 / 19002386.2134$

Dominguez-Lara, S. A. (2016c). Reporte de las diferencias confiables en el perfil del ACE-III. Neurología. doi: 10.1016/j.nrl.2016.02.022

Dominguez-Lara, S. (en revisión a). Prevalencia de procrastinación académica en estudiantes universitarios de Lima Metropolitana y su relación con variables demográficas.

Dominguez-Lara, S. (en revisión b). Valores normativos de una escala de autoeficacia académica en estudiantes universitarios de Lima.

Dominguez-Lara, S. (en revisión c). Inventario de Ansiedad ante Exámenes-Versión en español: diferencias según sexo y valores normativos en universitarios de Lima.

Dominguez-Lara, S. A., Merino-Soto, C., \& Navarro-Loli, J. (2016). Estimación paramétrica de la confiabilidad y diferencias confiables. Revista Médica de Chile, 144(3), 406-407. doi: 10.4067/S003498872016000300019

Dominguez-Lara, S. A., Villegas-García, G., \& Centeno-Leyva, S. B. (2014). Procrastinación académica: Validación de una escala en una muestra de estudiantes de una universidad privada. Liberabit, 20(2), 293-304. Recuperado de http://revistaliberabit.com/ es

Ellis, A., \& Knaus, W. J. (1977). Overcoming procrastination. New York: Institute for Rational Living.

Fernández-Arata, M., \& Merino-Soto, C. (2014). Error de medición alrededor de los puntos de corte en el MBI-GS. Liberabit, 20(2), 209-218. Recuperado de http://revistaliberabit.com/es

Furlan, L. A., Heredia, D. E., Piemontesi, S. E., \& Tuckman, B.W. (2012). Análisis factorial confirmatorio de la adaptación argentina de la escala de procrastinación de Tuckman (ATPS). Perspectivas en Psicología, 9(3), 142-149. Recuperado de http://www. redalyc.org/revista.oa? $\mathrm{id}=4835$

Furlan, L., Pérez, E., Moyano, M., \& Cassady, J. (2010). Propiedades psicométricas y estandarización de la Escala de Ansiedad Cognitiva frente a los Exámenes 
a la población universitaria argentina. Evaluar, 10, 22-31. Recuperado de https://revistas.unc.edu.ar/ index.php/revaluar/index

Ghasemi, A., \& Zahediasl, S. (2012). Normality tests for statistical analysis: A guide for non-statisticians. International Journal of Endocrinology and Metabolism, 10(2), 486-489. doi: 10.5812/ijem.3505

González-Brignardello, M. P., \& Sánchez-Elvira-Paniagua, A. (2013). ¿Puede amortiguar el engagement los efectos nocivos de la procrastinación académica? Acción Psicológica, 10(1), 117-134. doi: 10.5944/ ap.10.1.7039

Grunschel, C., Patrzek, J., \& Fries, S. (2013). Exploring reasons and consequences of academic procrastination: an interview study. European Journal of Psychology of Education, 28(3), 841-861. doi: 10.1007/ s10212-012-0143-4

Hancock, G. R., \& Mueller, R. O. (2001). Rethinking construct reliability within latent variable systems. En R. Cudeck, S. Du Toit, \& D. Sörbom (Eds.), Structural equation modeling: Past and present. A Festschrift in honor of Karl G. Jöreskog (pp. 195-216). Chicago, IL: Scientific Software International.

Harriot, J., \& Ferrari, J. R. (1996). Prevalence of procrastination among samples of adults. Psychological Reports, 78(2), 611-616. doi: 10.2466/ pr0.1996.78.2.611

Klassen, R. M., Krawchuk, L. L., \& Rajani, S. (2008). Academic procrastination of undergraduates: Low self-efficacy to self-regulate predicts higher levels of procrastination. Contemporary Educational Psychology, 33(4), 915-931. doi: 10.1016/j.cedpsych.2007.07.001

Klingsieck, K. B., Grund, A., Schmid, S., \& Fries, S. (2013). Why students procrastinate: A qualitative approach. Journal of College Student Development, 54(4), 397-412. doi: 10.1353/csd.2013.0060

Knaus, W. J. (2002). The Procrastination Workbook. USA: New Harbinger Publications.

Lay, C. H. (1986). At last, my research article on procrastination. Journal of Research in Personality, 20(4),
474-495. doi: 10.1016/0092-6566(86)90127-3

Lee, S.Y., Poon, W. Y., \& Bentler, P. M. (1995). A two-stage estimation of structural equation models with continuous and polytomous variables. British Journal of Mathematical and Statistical Psychology, 48(2), 339-358. doi: 10.1111/j.2044-8317.1995.tb01067.x

Livingston, S. A. (1972). A criterion-referenced application of classical test theory. Journal of Educational Measurement, 9(1), 13-26. doi: 10.1111/j.17453984.1972.tb00756.x

Malgady, R. G. (2007). How skewed are psychological data? A standardized index of effect size. The Journal of General Psychology, 134(3), 355-359. doi: 10.3200/GENP.134.3.355-360

McDonald, R. P. (1999). Test theory: A unified treatment. Mahwah, NJ: Lawrence Erlbaum Associates.

Merino-Soto, C., Navarro-Loli, J., \& García-Ramirez, W. (2014). Revisión de la consistencia interna del Inventario de Inteligencia Emocional de Bar-On, EQ-I: YV. Revista Peruana de Psicología y Trabajo Social, 3(1), 141-154. Recuperado de http://revistas. uigv.edu.pe/index.php/psicologia

Muñiz, J. (2003). Teoría clásica de los tests. Madrid: Pirámide.

Naveh-Benjamin, M., McKeachie, W. J., \& Lin, Y. G., (1987). Two types of test-anxious students: Support for an information processing model. Journal of Educational Psychology, 79(2), 131-136. doi: 10.1037/0022-0663.79.2.131

Özer, B. U. (2011). A cross sectional study on procrastination: Who procrastinate more? International Conference on Education, Research and Innovation, 18, 34-37.

Özer, B. U., Demir, A. \& Ferrari, J. R. (2009). Exploring academic procrastination among Turkish students: Possible gender differences in prevalence and reasons. Journal of Social Psychology, 149(2), 241-257. doi: 10.3200/SOCP.149.2.241-257

Pardo-Bolívar, D., Perilla-Ballesteros, L., \& Salinas-Ramírez, C. (2014). Relación entre procrastinación académica y ansiedad-rasgo en estudiantes de psico- 
logía. Cuadernos Hispanoamericanos de Psicología, 14(1), 31-44. Recuperado de http://revistas.unbosque.edu.co/index.php/CHP

Potts, T.J. (1987). Predicting procrastination on academic tasks with self-report personality measures (Tesis doctoral inédita) Hofstra University, New York.

Quant, D. M., \& Sánchez, A. (2012). Procrastinación, procrastinación académica: concepto e implicaciones. Revista Vanguardia Psicológica, 3(1), 45-59.

Ramírez-Benítez, Y., Jiménez-Morales, R. M., \& Díaz-Bringas, M. (2015). Matrices progresivas de Raven: Punto de corte para preescolares 4 - 6 años. Evaluar, 15, 123-133. Recuperado de https://revistas.unc.edu.ar/index.php/revaluar

Sánchez-Rosas, J. (2015). The Achievement Emotions Questionnaire-Argentine (AEQ-AR): Internal and external validity, reliability, gender differences and norm-referenced interpretation of test scores. Evaluar, 15, 41-74. Recuperado de https://revistas.unc. edu.ar/index.php/revaluar

Satorra, A., \& Bentler, P. M. (1994). Corrections to test statistics and standard errors in covariance structure analysis. En A. von Eye \& C. C. Clogg (Eds.), Latent variables analysis: Applications for developmental research (pp. 399-419). Thousand Oaks, CA: Sage.

Schraw, G., Wadkins, T., \& Olafson, L. (2007). Doing the things we do: A grounded theory of academic procrastination. Journal of Educational Psychology, 99(1), 12-25. doi: 10.1037/0022-0663.99.1.12

Serrano-Pintado, I., \& Escolar-Llamazares, M. C. (2014). Definición del constructo ansiedad ante los exámenes en estudiantes universitarios. Ansiedad y Estrés, 20(2-3), 165-180.

Serrano-Pintado, I., Sánchez-Mateos, J. D., \& Escolar-Llamazares, M. C. (2010). Eficacia diferencial de estrategias de afrontamiento en la reducción de la ansiedad ante los exámenes en función del tipo de variable principalmente afectada. Ansiedad y Estrés, 16(2-3), 109-126.

Serrano-Pintado, I., Sánchez-Mateos, J. D., \& Escolar-Llamazares, M. C. (2016). A stress inoculation program to cope with test anxiety: Differential efficacy as a function of worry or emotionality. Avances en Psicología Latinoamericana. 34(1), 3-18. doi: 10.12804/ ap134.1.2016.01

Solomon, L. J., \& Rothblum, E. D. (1984). Academic procrastination: Frequency and cognitive-behavioral correlates. Journal of Counseling Psychology, 31(4), 503-509. doi: 10.1037/0022-0167.31.4.503

Spielberger, C. D., \& Vagg, P. R. (1995). Test Anxiety: A transactional process model. En C. D. Spielberger\& P. R.Vagg (Eds.), Test Anxiety: Theory, assessment, and treatment (pp. 3-14). Washington, DC: Taylor \& Francis.

Steel, P. (2007). The nature of procrastination: A meta-analytic and theoretical review of quintessential self-regulatory failure. Psychological Bulletin, 133(1), 65-94. doi: 10.1037/0033-2909.133.1.65

Steel, P., \& Klingsieck, K. B. (2016). Academic procrastination: Psychological antecedents revisited. Australian Psychologist, 51(1), 36-46. doi: 10.1111/ ap. 12173

Tuckman, B. W. (1991). The development and concurrent validation of the Procrastination Scale. Educational and Psychological Measurement, 51(2), 473-480 doi: 10.1177/0013164491512022

Van Eerde, W. (2003). A meta-analytically derived nomological network of procrastination. Personality and Individual Differences, 35, 1401-1418. Recuperado de https://www.journals.elsevier.com/personality-and-individual-differences

Wäschle, K., Allgaier, A., Lachner, A., Fink, S., \& Nückles, M. (2014). Procrastination and self-efficacy: Tracing vicious and virtuous circles in self-regulated learning. Learning and Instruction, 29, 103-114. doi: 10.1016/j.learninstruc.2013.09.005

Wolters, C. A. (2003). Understanding procrastination from a self-regulated learning perspective. Journal of Educational Psychology, 95(1), 179-187. doi: 10.1037/0022-0663.95.1.179 